\title{
Antibacterial Activity of Polyethylenimine/Carrageenan Multilayer against Pathogenic Bacteria
}

\author{
Annabelle V. Briones ${ }^{*}$, Toshinori Sato², Ursela G. Bigol ${ }^{1}$ \\ ${ }^{1}$ Industrial Technology Development Institute, DOST Complex, Taguig, Philippines \\ ${ }^{2}$ Department of Biosciences and Informatics, Keio University, Yokohama, Japan \\ Email: *avbriones2003@yahoo.com
}

Received 1 January 2014; revised 1 February 2014; accepted 8 February 2014

Copyright (C) 2014 by authors and Scientific Research Publishing Inc.

This work is licensed under the Creative Commons Attribution International License (CC BY). http://creativecommons.org/licenses/by/4.0/

(c) (i) Open Access

\begin{abstract}
The multilayer of polyethylenimine (PEI) and carrageenan $(\kappa, \imath, \lambda)$ formed by layer-by-layer assembly was investigated for its antibacterial activity against Enterobacter cloaceae, Staphylococcus aureus and Enterococcus faecalis 29505 for potential use as coating on biomaterial surface. All the multilayers exhibited growth inhibition. PEI/Iota carrageenan multilayer was effective in inhibiting the growth of the E. cloaceae, S. aureus and E. faecalis while PEI/Lambda carrageenan was effective in inhibiting the growth of $E$. cloaceae. Results of the paper strip test for combined action of carrageenan and PEI showed synergism with regards to bacterial growth inhibition. The multilayers had also contact-killing effect with the test organisms. The multilayer was also characterized by atomic force microscopy (AFM), X-ray photoelectron spectroscopy (XPS) and biomolecular interaction analysis.
\end{abstract}

\section{Keywords}

Carrageenan, Polyethylenimine, Bacterial Growth Inhibition, Anti-Bacterial

\section{Introduction}

Designing an antibacterial coating on biomaterial surfaces using layer-by-layer technique is becoming popular. This technique is based on alternating deposition of oppositely charged synthetic or natural polyelectrolytes to form a thin film layer-by-layer assembly which is a cheap method eliminating complicated multistage covalent grafting process that produces materials with controllable adhesive properties [1]-[3]. There are a lot of antibac-

${ }^{*}$ Corresponding author.

How to cite this paper: Briones, A.V., et al. (2014) Antibacterial Activity of Polyethylenimine/Carrageenan Multilayer against Pathogenic Bacteria. Advances in Chemical Engineering and Science, 4, 233-241. 
terial coatings developed based on hydrophobicing agents, application of positive charge, polymer brush coatings [4] [5], thermoresponsive polymers [6], drug eluting coatings, silver coatings, natural [7] or synthetic polymeric biocides [8] [9]. However, clinical efficacies of these many coatings still have to be validated. Coatings should not negatively impact tissue integration of a prosthesis which would be the best guarantee for a long-term functioning of an implanted prosthesis [10].

Previous studies done using this technique were the use of chitosan/hyaluronan and chitosan/ $\kappa$-carrageenan multilayers [11]. In this study, polyethylenimine (Figure 1) was used instead of chitosan then layered with the three types of carrageenan (Figure 1). There have been no studies reported yet on the use of PEI-carrageenan multilayer for use as antibacterial coating without modifying the structure of PEI. Hence, the aim of this study is to evaluate the potential of PEI-carrageenan multilayer as antibacterial coating.

Polyethylenimine (PEI) is a weakly basic, aliphatic, nontoxic synthetic polymer which is polycationic owing to the presence of primary, secondary, and tertiary amino groups [12]. It has a strong permeabilizing effect but no bactericidal effect on gram-negative bacteria [13]. PEI prepared in nanoparticles was incorporated in dental provisional cements and found to had antibacterial effect against Streptococcus mutans [14]. Insoluble crosslinked quaternary ammonium polyethylenimine (PEI) nanoparticles incorporated at $1 \mathrm{or} 2 \% \mathrm{w} / \mathrm{w}$ in a resin composite also have an antimicrobial effect against Staphylococcus aureus, Staphylococcus epidermidis, Enterococcus faecalis, Pseudomonas aeruginosa and Escherichia coli [15].

Carrageenan is classified as a food additive [16] and shows the potential use for a new drug delivery system providing more control over the release rate of drugs. Being a sulfated polysaccharide, carrageenan is biocompatible, biodegradable, nontoxic, cheap and gel forming [17], however, the use of carrageenan as antibacterial coating is limited [18] [19]. In one study, oligosaccharides from $\kappa$-carrageenan have antibacterial activities against E. coli, S. aureus, S. cere, P. citr and Mucor sp. [20].

\section{Experimental}

\subsection{Materials}

PEI (average MW = $25 \mathrm{KDA}$, branched with degree of polymerization of 580) was obtained from Aldrich Company (St Quentin Fallavier, France). $\kappa$-carrageenan (Bengel KK-100, Lot No. XO300-2), $l$-carrageenan (Benvisco SI-100, Lot No.M1400-1) and $\lambda$-carrageenan (Benvisco SL-100, Lot No. S2703-2) was obtained from Shemberg Biotech Corporation (Carmen, Cebu, Philippines, 6005). Dulbecco's PBS (-) was obtained from Nissui Pharmaceutical Co. Ltd (Japan).

\subsection{Sample Preparation}

$\kappa, l, \lambda$ carrageenan and PEI were dissolved in PBS (-) solution then mixed using a vortex machine, followed by

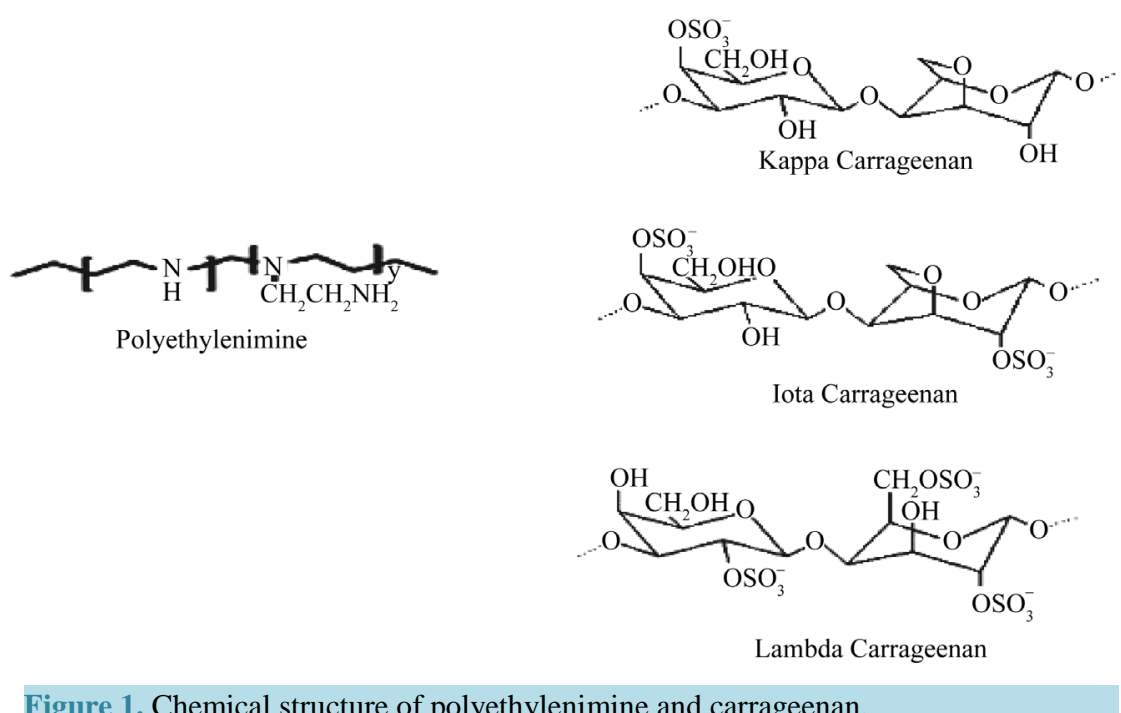

Figure 1. Chemical structure of polyethylenimine and carrageenan. 
ultrasonication and shaken at 200 rpm for 8 hours. Final concentration of each sample is: $1 \mathrm{mg} / \mathrm{mL}$.

\subsection{Preparation of Multilayers}

Multilayer was prepared by successive immersion of a substratum to solutions of carrageenan and PEI for 10 minutes then allowed to dry at room temperature followed by washing with water to remove excess buffer solution and allowed to dry again. This was done alternately for each polymer. The process was repeated thrice each for every solution to make a final of 6 coatings with alternating layer of carrageenan and PEI solution.

\subsection{Atomic Force Microscopy (AFM) Measurement}

This method was previously published in International Proceedings of Chemical, Biological, Environmental Engineering Journal [21]. Imaging was done through a commercial SPA-300 system of Seiko Instruments, Inc. Japan. A Si3N4 tip on the cantilever with a length of $100 \mathrm{~nm}$ and a depth of $400 \mathrm{~nm}$ (SN-AF01-A, Olympus Optical Co.) was used with tapping mode. The imaging was recorded in non-contact mode under ambient condition. Solutions of PEI and carrageenan on the mica disk were observed as control.

\subsection{Biomolecular Interaction Analysis}

This method was previously published in International Proceedings of Chemical, Biological, Environmental Engineering Journal [21]. Analysis of biomolecular binding of carrageenan and PEI was done using BIACORE X, Pharmacia Biosensor AB, Uppsala, Sweden equipped with sensor chip C1 BIACORE Lot no. 10020781. Samples of $\kappa, \imath, \lambda$-carrageenan solution and PEI solution at $1 \mathrm{mg} / \mathrm{mL}$ concentration was injected at $120 \mathrm{uL}$ with a flowrate of $10 \mathrm{uL} / \mathrm{min}$.

\subsection{Surface Composition of the Multilayer by X-Ray Photoelectron Spectroscopy (XPS)}

The surface composition of the multilayer was measured using X-ray photoelectron spectroscopy (XPS), model JPS-9000MX (JEOL) using Mg $\mathrm{K}_{\alpha}$ radiation (10 kV-10 mA). Peak positions were calibrated $\mathrm{C}_{1 \mathrm{~s}}$ position at 284 $\mathrm{eV}$.

\subsection{Bacterial Growth Inhibition}

Antibacterial activity was determined using the methodology of S.G. Hu et al. [22], with some modification. The bacteria used in this study were: E. cloaceace, S. aureaus and E. faecalis. Frozen preserved stock was thawed at room temperature, and then $0.1 \mathrm{~mL}$ was pipetted and streaked into a quadrant on a sheep-blood agar plate (Difco Laboratories, Detroit, MI) and cultured at $37^{\circ} \mathrm{C}$ overnight. Afterward, a single colony was scraped with a loop and swabbed onto a $15^{\circ}$-slant medium $\left(10 \mathrm{~mL}\right.$ of nutrient agar) and was incubated at $37^{\circ} \mathrm{C}$. After 18 - $24 \mathrm{~h}$ of culturing, $20 \mathrm{~mL}$ of PBS was added $\left(72 \mathrm{~mL}\right.$ of $0.2 \mathrm{M} \mathrm{Na} \mathrm{NPO}_{4}$ was mixed with $28 \mathrm{~mL}$ of $0.2 \mathrm{M}$ $\mathrm{NaH}_{2} \mathrm{PO}_{4}$, and $0.5 \mathrm{~g}$ of $\mathrm{NaCl}$ and $2 \mathrm{~g} / \mathrm{L}$ Tween 80 were added; this brought the total volume to $1000 \mathrm{~mL}$ ). After mixing, $1 \mathrm{~mL}$ of the solution was moved into $9 \mathrm{~mL}$ of nutrient broth (concentration $=8 \mathrm{~g} / \mathrm{L}$ ) and mixed with a vortex mixer. The solution was then diluted with PBS to $(1.5 \pm 0.3) \times 10^{5}$ cell/ $\mathrm{mL}$ and was placed in flasks (three samples, with PEI/Carrageenan coated mica disk per sample for each group). After incubation at $37^{\circ} \mathrm{C}$ for 0 - $18 \mathrm{~h}, 20 \mathrm{~mL}$ of PBS was added, and the mixture was stirred for $30 \mathrm{~s}$. Consecutive dilute solutions were prepared by $1 \mathrm{~mL}$ of the previous solution being mixed with $9 \mathrm{~mL}$ of PBS. From this solution, $1 \mathrm{~mL}$ was transferred to a $50-\mathrm{mL}$ centrifugal tube, mixed with $15 \mathrm{~mL}$ of nutrient agar (at $45^{\circ} \mathrm{C}$ ), poured into a 9-cm plate, allowed to cool, and incubated at $37^{\circ} \mathrm{C}$ for 18 - $24 \mathrm{~h}$. The number of surviving bacteria was then counted.

\subsection{Paper Strip Test for Combined Action}

The nutrient agar was prepared by dissolving $2.3 \mathrm{~g}$ in $100 \mathrm{~mL}$ of water and autoclaved for 15 minutes at $121^{\circ} \mathrm{C}$. After sterilization, $10 \mathrm{~mL}$ was plated and allowed to solidify. The sterilized agar medium has been seeded with the test organism in the same manner as in the agar-diffusion test. One of the strip was coated with PEI and placed vertically on the plate while the other strip coated with carrageenan and multilayers of PEI/Carrageenan was placed horizontally. 


\subsection{Zone of Inhibition}

The nutrient agar was prepared as of the preparation of the paper strip test. Test organisms were streaked singly and continuously in the sterilized agar media parallel to each other. Test strips coated with PEI/Carrageenan were placed on top of the media crossing the streaked line.

\section{Results and Discussion}

\subsection{Formation of Multilayers}

The multilayer was formed by electrostatic interaction between the cationic PEI and anionic carragenan using the layer-by-layer self-assembly technique [23] [24]. The interaction of PEI and carrageenan was due to the opposite charges between the positively charged amine groups $\left(\mathrm{NH}_{3}\right)$ in PEI and the negatively charged sulfate groups $\left(\mathrm{SO}_{4}\right)$ in carrageenan. This method is very useful in many applications that require multilayer thin films. The process is relatively inexpensive with no complex reaction mechanisms. The process is simple just to dip a substrate into alternating positive and negative charge containing solutions to form uniform and stable layers.

The formation and build-up of multilayers were monitored using an atomic force microscope (AFM, SPA-300 system of Seiko Instruments, Inc. Japan). The results were previously published in International Proceedings of Chemical, Biological, Environmental Engineering Journal [21]. The AFM micrographs of the PEI/carrageenan bi-layer showed the presence of nanorings confirming the formation of layer-by-layer assembly [21]. The AFM micrographs also showed the change in height of the bi-layer film as compared to the images of single polymers (Carrageenan and PEI) [21]. The formation of bi-layer was further analyzed using a biosensor (BIACORE X). The results were also previously published in International Proceedings of Chemical, Biological, Environmental Engineering Journal [21]. The results showed that PEI interacted molecularly with carrageenan with PEI [21]. Binding capacity $\left(\mathrm{pg} / \mathrm{mm}^{2}\right.$ ) for each type of carrageenan with PEI was also shown [21]. The multilayer of $\mathrm{PEI} /$ Lambda carrageenan had the highest binding capacity of $6074.24 \mathrm{pg} / \mathrm{mm}^{2}$ followed by PEI/Kappa carrageenan $\left(1916.08 \mathrm{pg} / \mathrm{mm}^{2}\right)$ and PEI/Iota carrageenan multilayers $\left(1844.1 \mathrm{pg} / \mathrm{mm}^{2}\right)$ [21]. Based on the binding curves of all multilayers [21], a single injection of PEI apparently reached binding equilibrium with the membrane. Then after injection with carrageenan the signal increased, reaching a higher plateau confirming binding of the two polymers as observed in the staircase-like binding curves in all multilayers [21].

\subsection{Surface Composition of the Multilayer by XPS}

The XPS survey spectra of the multilayer of carrageenan and polyethylenimine is shown in Figure 2. All multilayers showed similar spectra regardless of the mode of deposition. XPS is one of the most commonly used techniques of surface analysis [25]. Upon exposure of the sample to an X-ray beam, the binding energies of characteristically emitted photoelectrons are measured, providing information on the elements from which they originate, as well as their chemical bonding [25]. As shown in Figure 3, all spectra did not show peaks at 285.0 $\mathrm{eV}$ which represent saturated hydrocarbons suggesting that no carbon contamination for all the surfaces. The surfaces of PEI/Iota Carrageenan and PEI/Lambda Carrageenan have peaks in $286.8 \mathrm{eV}$ and $286.6 \mathrm{eV}$ (Figure 3) respectively due to C-N groups of PEI and the residual amide groups while PEI/Kappa carrageenan tend to shift to the lower side and has a peak at $287.2 \mathrm{eV}$. The peak observed at 287.5 for both PEI/Iota Carrageenan and $\mathrm{PEI} /$ Lambda Carrageenan is due to the O-C-O groups of the saccharide unit. The O-C-O groups of PEI/Kappa carrageenan is observed at $288.2 \mathrm{eV}$. Among the three surfaces, PEI/Lambda Carrageenan showed a peak at $288.4 \mathrm{eV}$ due to oxidized saccharide species.

\subsection{Bacterial Growth Inhibition}

The results of bacterial growth inhibition are shown in Figures 4-6. All the multilayers exhibited growth inhibition. The multilayer of PEI/Lambda carrageenan showed the highest growth inhibition of E. cloaceae of $84.6 \%$ followed by PEI/Iota carrageenan of $81.32 \%$ and PEI/Kappa carrageenan of $69.12 \%$ (Figure 4). Against S. aureus the multilayer of PEI/Iota carrageenan showed the highest inhibition growth of $66.84 \%$ followed by PEI/Kappa carrageenan of 38.86\% and PEI/Lambda carrageenan of 38.35\% (Figure 5). The multilayers were also tried against E. faecalis. This organism is known to be involved in the clogging of biliary stents [1]. Again the multilayer of PEI/Iota carrageenan showed the highest inhibition growth of $77.66 \%$ followed by PEI/Kappa 


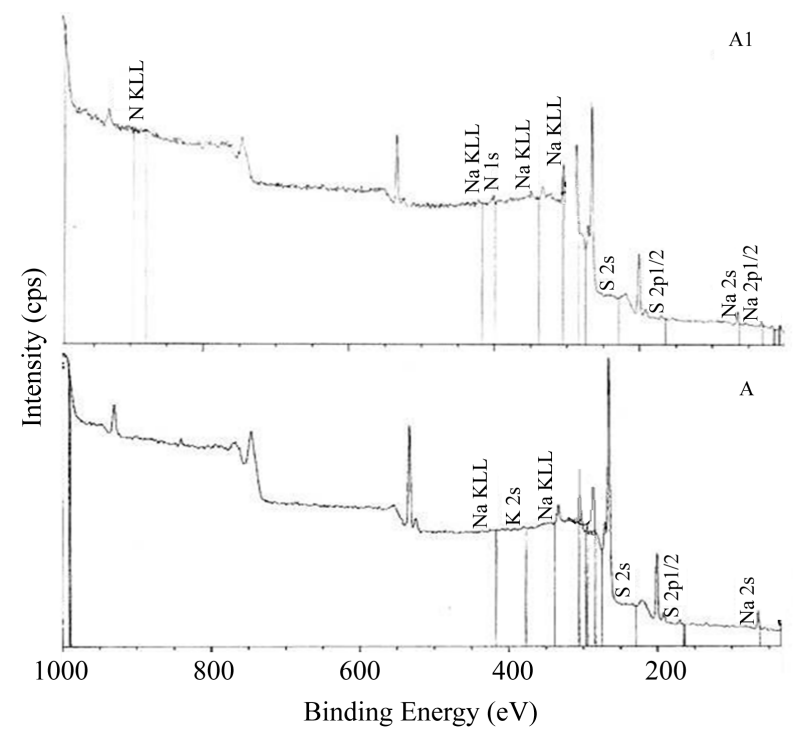

A1

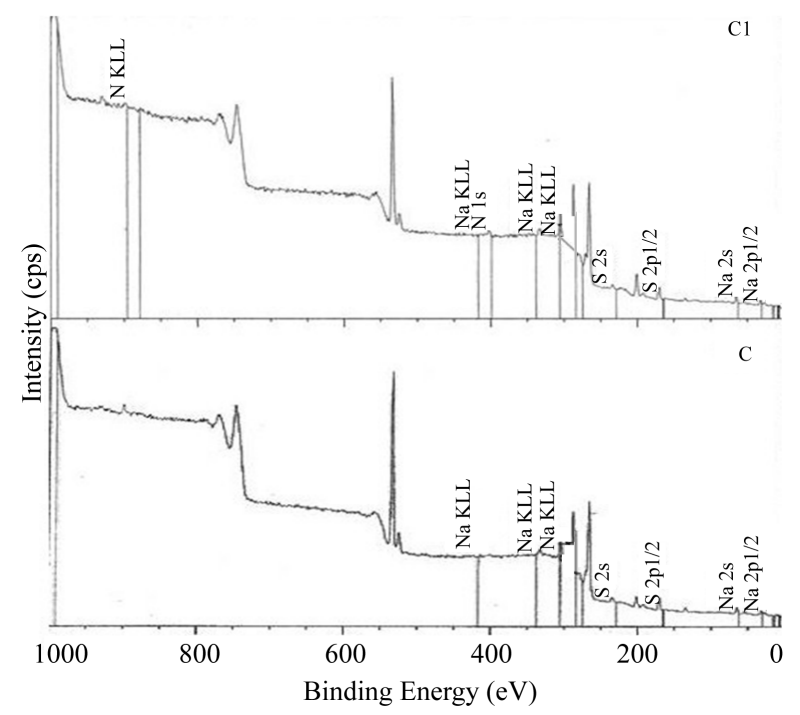

Figure 2. XPS spectra of multilayers of PEI/Carrageenan. A. Kappa carrageenan, B. Iota carrageenan, C. Lambda carrageenan, A1. PEI/Kappa carrageenan, B1. PEI/Iota carrageenan, C1. PEI/Lambda carrageenan.

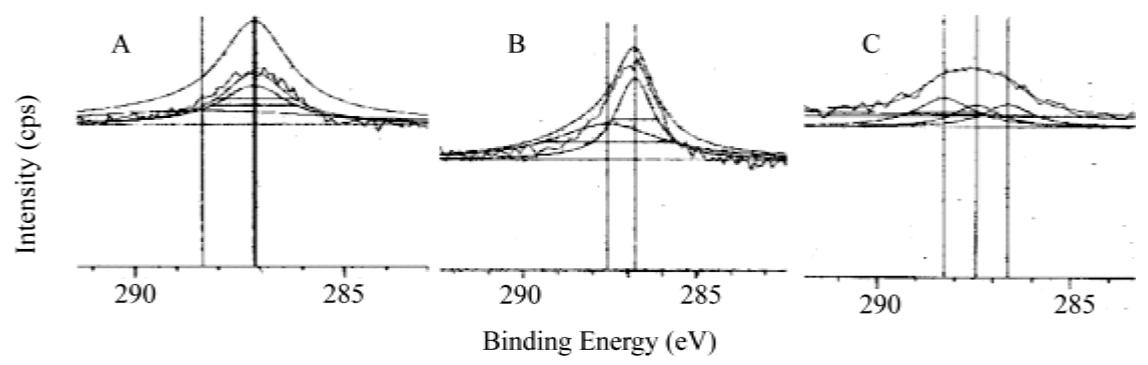

Figure 3. XPS carbon spectra of multilayer. A. PEI/Kappa carrageenan, B. PEI/Iota carrageenan, C. PEI/Lambda carrageenan.

carrageenan of $62.17 \%$ and PEI/Lambda carrageenan of 57.28\% (Figure 6). In summary, PEI/Iota carrageenan is effective in inhibiting the growth of the E. cloaceae, S. aureus and E. faecalis while PEI/Lambda carrageenan is effective in inhibiting the growth of $E$. cloaceae. The PEI and carrageenan alone were also investigated for its 


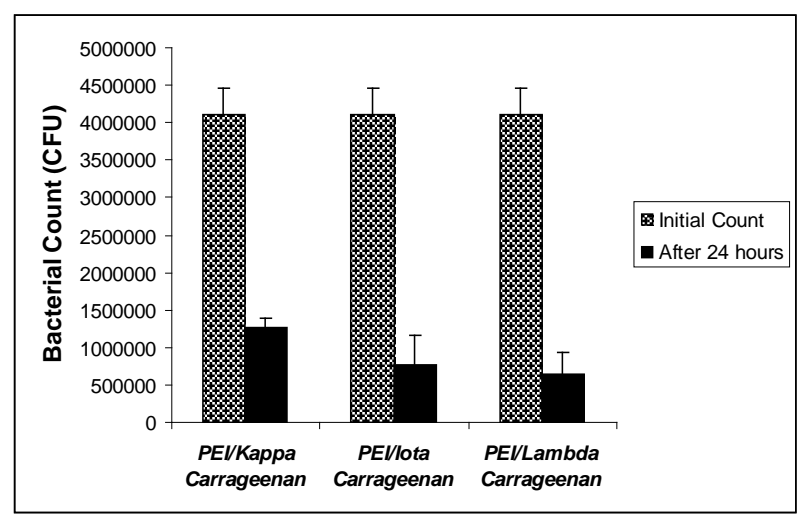

Figure 4. Bacterial growth of Enterobacter cloaceae after 24 hours exposure to multilayers of PEI and carrageenan.

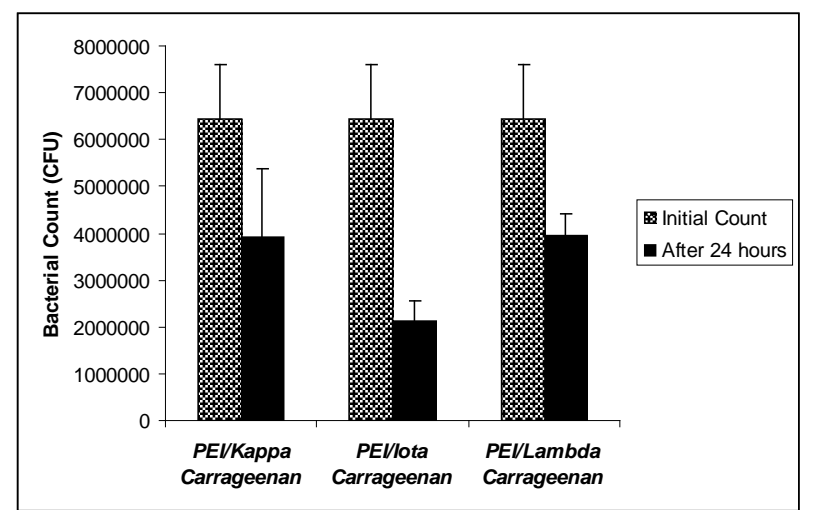

Figure 5. Bacterial growth of Staphylococcus aureus after 24 hours exposure to multilayers of PEI and carrageenan.

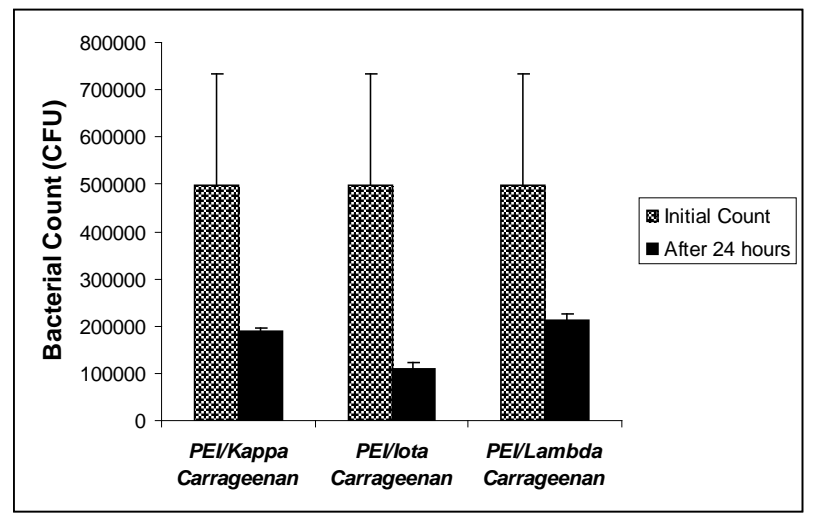

Figure 6. Bacterial growth of Enterococcus faecalis (29505) after 24 hours exposure to multilayers of PEI and carrageenan.

ability to inhibit bacterial growth. Shown in Figure 7 are the results of bacterial count after 24 hours incubation period with PEI and carrageenan. PEI has zero bacterial growth (100\% growth inhibition) when exposed to $S$. aureus and E. cloaceace (Figure 7) but when exposed to E. faecalis, inhibition growth is only about $76.2 \%$ (Figure 7). Carrageenan showed bacterial growth inhibition in S. aureus with the highest inhibition growth of 25.0\% by lambda carrageenan (Figure 7) while iota carrageenan displayed the highest inhibition growth of $48.20 \%$ to E. faecalis and $4.1 \%$ inhibition growth to E. cloaceae. Lambda carrageenan did not show any inhibi- 


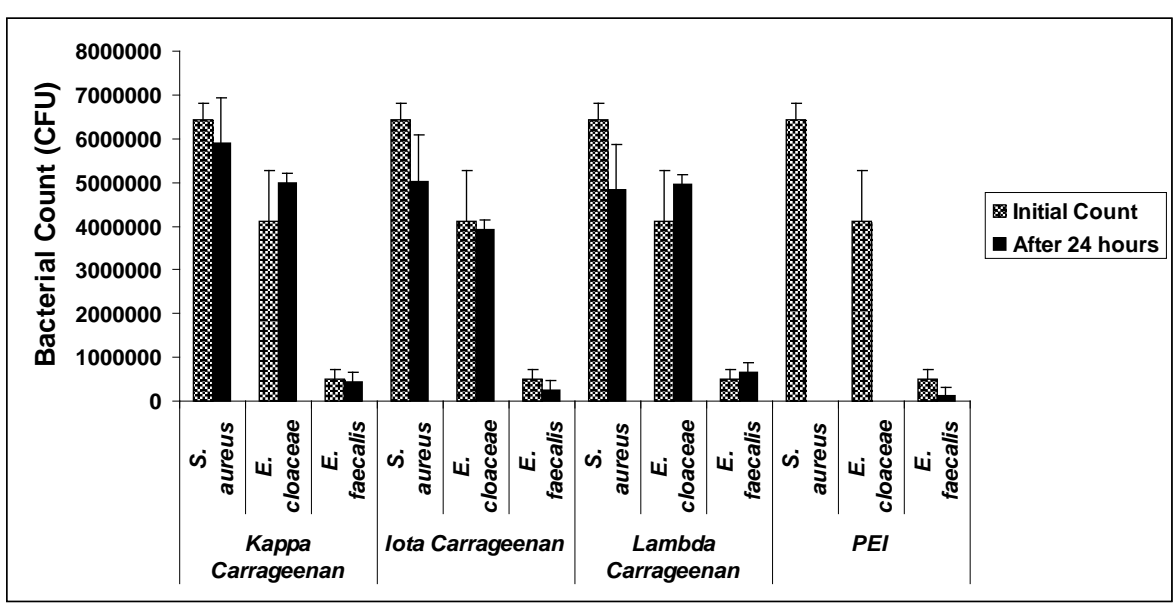

Figure 7. Bacterial growth of Enterococcus faecalis (29505) after 24 hours exposure to PEI and carrageenan.

tion growth for both E. cloaceae and E. faecalis (Figure 7) instead it increased the growth of E. faecalis by 29.5\% (Figure 7). Kappa carrageenan and lambda carrageenan showed an increase of growth in E. cloaceae by $18.0 \%$ and $17.44 \%$ respectively (Figure 7). Although the results of bacterial growth inhibition obtained from carrageenan were not that quite high for $S$. aureus, it indeed showed that it has antibacterial activity as previously reported [20].

\subsection{Paper Strip Test for Combined Action}

Figure 8, Figure 9 showed the paper strip test for combined action of double layer and multilayers of carrageenan and PEI. The test was done to determine the antagonizing effect of PEI with carrageenan. During incubation PEI diffused together with carrageenan and the shape of inhibition area within the area of overlap indicates whether stimulation or antagonism has taken place. The results showed that PEI and carrageenan have synergistic effect with regards to antibacterial activity and does not antagonize nor create competitive reversal of bacterial growth inhibition of $S$. aureus and E. cloaceae. Synergism is displayed by a clear area devoid of growth in the angle between PEI (vertical) and carrageenan (horizontal) strips as observed in both Figure 8, Figure 9. Lack of synergism is displayed by growth of organism in the zone of inhibition which is not observed in both figures.

\subsection{Zone of Inhibition Test}

The result of zone of inhibition test is found in Figure 10. The multilayers of PEI/Carrageenan were tested against E. coli, E. cloaceae and S. aureus. The zone of inhibition was observed after 24 and 48 hours. As shown in Figure 10(a), there was no growth observed for all the test strips after 24 hours of incubation. Extending the incubation to 48 hours of exposure, still no growth was observed and the zone of inhibition is more distinct than overnight incubation as observed in S. aureus (Figure 10(b)). The test clearly showed that the multilayers of $\mathrm{PEI} /$ Carrageenan have contact-killing effect with the test organisms.

\section{Summary and Conclusion}

The study showed that multilayer of carrageenan/polyethyleimine was formed using the layer-by-layer assembly absorption technique. The formation was monitored by atomic force microscopy (AFM) and biomolecular interaction analysis. PEI interacted molecularly with carrageenan to form the multilayer. The multilayer showed antibacterial activity against Enterobacter cloaceae, Staphylococcus aureus and Enterococcus faecalis. PEI/Iota carrageenan is effective in inhibiting the growth of $S$. aureus and E. faecalis while $\mathrm{PEI} / L a m b d a$ Carrageenan is most effective in inhibiting the growth of E. cloaceae. PEI/Kappa Carrageenan is effective in inhibiting the growth of E. faecalis. All multilayers have contact-killing effects to the test organisms. Synergism was also observed between PEI and carrageeenan for its combined action on antibacterial growth. 


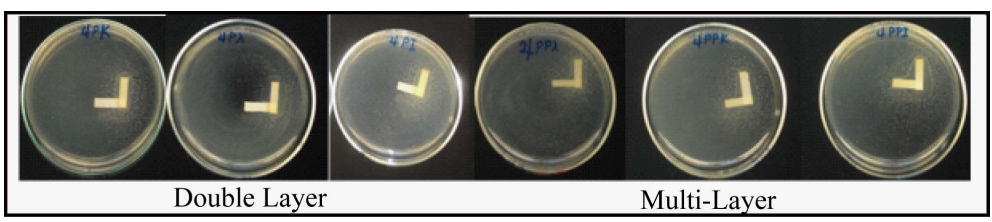

Figure 8. Paper strip test for combined action of the Double and Multilayers of Carrageenan and Polyethylenimine in S. aureus. A \& E. PEI/Kappa carrageenan. B \& D. PEI/Lambda carrageenan. C \& F. PEI/Iota carrageenan. All experiments were done in triplicate.

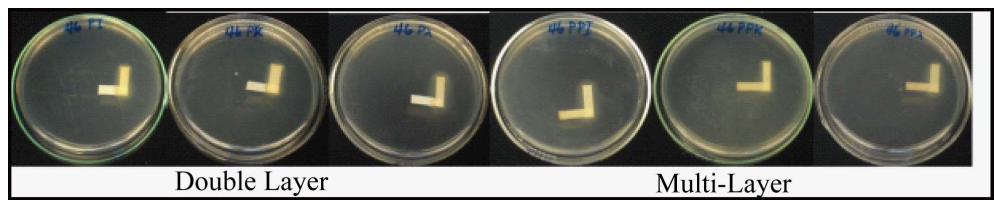

Figure 9. Paper strip test for combined action of the Double andMultilayers of Carrageenan and Polyethylenimine in E. cloaceae. A \& D. PEI/Iota carrageenan. B \& E. PEI/Kappa carrageenan. C \& F. PEI/Lambda carrageenan. All experiments were done in triplicate.

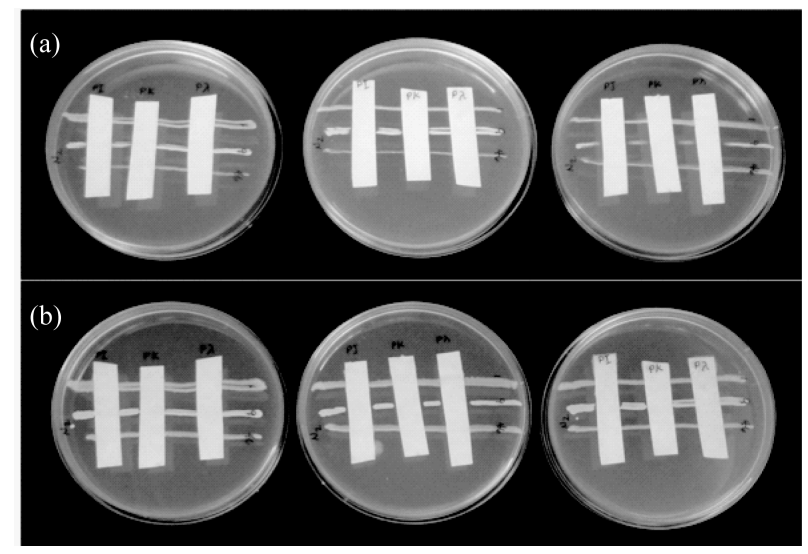

Figure 10. Zone inhibition test against E. coli (1), E. cloaceae (46) and S. aureus (9). (a) 24 hours incubation; (b) 48 hours incubation. All experiments were done in triplicate.

\section{Acknowledgements}

Sincere thanks to Japan Society for the Promotion of Science (JSPS) for the financial support from the grant under the RONPAKU dissertation program and Keio University, as well as to the Industrial Technology Development Institute, and the Department of Science \& Technology, Manila, Philippines.

\section{References}

[1] Mendelson, J.D., Yang, S.Y., Hiller, J., Hochbaum, A.I. and Rubner, M.F. (2003) Rational Design of Cytophilic and Cytophobic Polyelectrolyte Multilayer Thin Films. Biomacromolecules, 4, 96-106. http://dx.doi.org/10.1021/bm0256101

[2] Elbert, D.L., Herbert, C.B. and Hubbell, J.A. (1999) Thin Polymer Layers Formed by Polyelectrolyte Multilayer Techniques on Biological Surfaces. Langmuir, 15, 5355-5362. http://dx.doi.org/10.1021/la9815749

[3] Richert, L., Lavalle, P., Payan, E., Shu, X., Prestwich, G., Stoltz, J., Schaaf, P., Voegel, J. and Picart, C. (2004) Layer by Layer Buildup of Polysaccharide Films: Physical Chemistry and Cellular Adhesion Aspects. Langmuir, 20, 448-458. http://dx.doi.org/10.1021/la035415n

[4] Sharma, S., Johnson, R.W. and Desai, T.A. (2004) Evaluation of the Stability of Nonfouling Ultrathin Poly(ethylene glycol) Films for Silicon-Based Micro Devices. Langmuir, 20, 348-356. http://dx.doi.org/10.1021/la0347531 
[5] Roosjen, A., Van der Mei, H.C., Busscher, H.J. and Norde, W. (2004) Microbial Adhesion to Poly(ethylene oxide) Brushes: Influence of Polymer Chain Length and Temperature. Langmuir, 20, 10949-55. http://dx.doi.org/10.1021/la048469l

[6] Yamato, M., Konno, C., Utsumi, M., Kikuchi, A. and Okano, T. (2002) Thermally Responsive Polymer-Grafted Surfaces Facilitate Patterned Cell Seeding and Co-Culture. Biomaterials, 23, 561-567. http://dx.doi.org/10.1016/S0142-9612(01)00138-7

[7] Hu, S.G., Jou, C.H. and Yang, M.C. (2002) Surface Grafting of Polyester Fiber. Journal of Applied Polymer Science, 86, 2977-2983. http://dx.doi.org/10.1002/app.11261

[8] Cen, L., Neoh, K.G. and Kang, E.T. (2003) Surface Functionalization Techniques for Conferring Anti Bacterial Properties to Polymeric and Cellulosic Surfaces. Langmuir, 19, 10295-10303. http://dx.doi.org/10.1021/la035104c

[9] Kanazawa, A., Ikeda, T. and Endo, T. (1994) Polymeric Phosphonium Salts as a Novel Class of Cationic Biocides. VI. Antibacterial Activity of Fibers Surface-Treated with Phosphonium Salts Containing Trimethoxysilane Groups. Journal of Applied Polymer Science, 52, 641-647. http://dx.doi.org/10.1002/app.1994.070520507

[10] Gristina, A.G. (1987) Biomaterial-Centered Infection: Microbial Adhesion versus Tissue Integration. Science, 237, 1588-1595. http://dx.doi.org/10.1126/science.3629258

[11] Bratskaya, S., Marinin, D., Simon, F., Synytska, A., Zschoche, S., Busscher, H.J., Jager, D. and van der Mei, H.C. (2007) Adhesion and Viability of Two Enterococcal Strains on Covalently Grafted Chitosan and Chitosan/k-Carrageenan Multilayers. Biomacro Molecules, 8, 2960-2968. http://dx.doi.org/10.1021/bm700620j

[12] Khalil, H., Chen, T., Riffon, R., Wang, R. and Wang, Z. (2008) Synergy between Polyethylenimine and Different Families of Antibiotics against a Resistant Clinical Isolate of Pseudomonas aeruginosa. Antimicrobial Agents and Chemotherapy, 52, 1635-1641. http://dx.doi.org/10.1128/AAC.01071-07

[13] Helander, I.M., Alakomi, H., Latva-Kala, K. and Koski, P. (1997) Polyethylenimine Is an Effective Permeabilizer of Gram-Negative Bacteria. Microbiology, 143, 3193-3199. http://dx.doi.org/10.1099/00221287-143-10-3193

[14] Shvero, D.K., Davidi, M.P., Weiss, E.I., Srerer, N. and Beyth, N. (2010) Antibacterial Effect of Polyethyleneimine Nanoparticles Incorporated in Provisional Cements against Streptococcus mutans. Journal of Biomedical Materials Research Part B: Applied Biomaterials, 94, 367-371. http://dx.doi.org/10.1002/jbm.b.31662

[15] Beyth, N., Houri-Haddad, Y., Baraness-Hadar, L., Yudovin-Farber, I., Domb, A.J. and Weiss, E.I. (2008) Surface Antimicrobial Activity and Biocompatibility of Incorporated Polyethylenimine Nanoparticles. Biomaterials, 29, 41574163. http://dx.doi.org/10.1016/j.biomaterials.2008.07.003

[16] (2007) Evaluation of Certain Food Additives and Contaminants. Sixty-Eighth Report of the Joint FAO/WHO Expert Committee on Food Additives. WHO Technical Report Series 947, 32.

[17] Richardson, R.K. and Goycoolea, F.M. (1994) Rheological Measurement of k-Carrageenan during Gelation. Carbohydrate Polymer, 24, 223-225. http://dx.doi.org/10.1016/0144-8617(94)90134-1

[18] Schoeler, B., Delorme, N., Doench, I., Sukhorukov, G.B., Fery, A. and Glinel, K. (2003) Polyelectrolyte Films Based on Polysaccharides of Different Confrmations: Effects on Multilayer Structure and Mechanical Properties. Biomacromolecules, 7, 2065-2071. http://dx.doi.org/10.1021/bm060378a

[19] Lee, S.S., Lee, K.B. and Hong, J.D. (2003) Evidence for Spin Coating Electrostatic Self-Assembly of Polyelectrolytes. Langmuir, 19, 7592-7596. http://dx.doi.org/10.1021/la034263t

[20] Wang, F.F., Yao, Z., Wu, H.G., Zhang, S.X., Zhu, N.N. and Gai, X. (2011) Antibacterial Activities of Kappa-Carrageenan Oligosaccharides. Applied Mechanics and Materials, 108, 194-199.

[21] Briones, A.V. and Sato, T. (2011) Formation of Polyethylenimine/Carrageenan Bi-Layer as Monitored by Atomic Force Microscopy and Biomolecular Interaction Analysis. International Proceedings of Chemical, Biological and Environmental Engineering Journal, 24, 288-291. http://dx.doi.org/10.7763/ipcbee

[22] Hu, S.G., Jou, C.H. and Yang, M.C. (2002) Antibacterial and Biodegradable Properties of Polyhydroxyalkanoates Grafted with Chitosan and Chitooligosaccharides via Ozone Treatment. Journal of Applied Polymer Science, 86, 29772983. http://dx.doi.org/10.1002/app.12055

[23] Decher, G. (1997) Fuzzy Nanoassemblies: Toward Layered Polymeric Multicomposites. Science, 277, 1232-1237. http://dx.doi.org/10.1126/science.277.5330.1232

[24] Decher, G., Hong, J.D. and Schmitt, J. (1992) Buildup of Ultrathin Multilayer Films by A Self-Assembly Process. III. Consecutively Alternating Adsorption of Anionic and Cationic Polyelectrolytes on Charged Surfaces. Thin Solid Films, 210-211, 831-835. http://dx.doi.org/10.1016/0040-6090(92)90417-A

[25] Rodrigues, S., Rosa da Costa, A.M. and Grenha, A. (2012) Chitosan/Carrageenan Nanoparticles: Effect of CrossLinking with Tripolyphosphate and Charge Ratios. Carbohydrate Polymers, 89, 282-289. http://dx.doi.org/10.1016/j.carbpol.2012.03.010 